\title{
Algunas consideraciones sobre la propuesta editorial para la enseñanza de Historia Antigua
}

Andrea Paula Zingarelli

Para entender la necesidad de reflexionar sobre el material de lectura con el que contamos para el proceso de enseñanza aprendizaje de la historia antigua debemos referirnos a nuestra práctica cotidiana, donde ningún docente puede escapar a la utilización de manuales.

Un análisis del uso de manuales en las distintas épocas es una mirada a la concepción de la historia de ese momento, a los programas de estudio y también a nuestra práctica docente.

El punto de partida de este trabajo será por un lado mirar hacia atrás y bucear en la tradición ${ }^{1}$ escolar; por otro, ver si existe actualmente correspondencia entre los nuevos enfoques didácticos y la propuesta de las editoriales y por último, preguntarnos si se puede ofrecer una interpretación de textos alternativos al manual, que permitan al alumno acercarse a las nuevas problemáticas de la disciplina en el área de la historia antigua.

Varias generaciones de estudiantes secundarios *hemos aprendido* la historia de los manuales de Historia Antigua y Medieval de Ibañez ${ }^{2}$, Drago ${ }^{3}$, que formaban parte de los enfoques tradicionales de la enseñanza aprendizaje de la historia y donde se mantenían fuertemente arraigadas las prácticas positivistas.

La historia fue entendida durante mucho tiempo como relato erudito, es decir, se limitaba a ordenar hechos considerados únicos e irrepetibles y, como dice Tuñon de Lara ${ }^{4}$, nunca pudo ser plenamente inocente: la recuperación del pasado fue una justificación del mismo.

De este modo los contenidos de los libros de textos incluían, ordenadas cronológicamente, las acciones de los hombres, no la memoria colectiva de todo un pueblo 5 sino la acumulación de datos del pasado político institucional. Ejemplo de esto encontramos

* El enfoque del tema se limita a la historia del Cercano Oriente Antiguo ya que la autora se dedica a esa área. 
en el listado de los faraones que gobernaron Egipto y la duración de su reinado durante el Imperio Nuevo ${ }^{6}$, la descripción de los rasgos físicos de los súmeros y las razones por las que alcanzaron un alto grado de civilización: «eran hombres de baja estatura, gruesos, de cabeza redonda, y rapada, ojos salientes y nariz grande (...) Debido a su inteligencia y laboriosidad y favorecidos por el medio geográfico (...)».7

Existen también trabajos ${ }^{8}$ como la Historia Antigua y Medieval de J. L. Romero que, sin dejar de ser de recopilación de datos y ordenamiento cronológico de los mismos, tratan de dar algunas explicaciones sobre los cambios sociales. No conciben una historia meramente anecdótica centrada en los hechos más puntuales y los personajes más destacados, sino que dan cuenta de las estructuras socioeconómicas del mundo antiguo. ${ }^{9}$

Otro rasgo de la mayoría de los libros de texto son las explicaciones causales. En el manual de la editorial A. ZETA ${ }^{10}$ en el capítulo que trata sobre conocimiento histórico se expresa la idea de que la historia es una ciencia social que intenta explicar las causas y las consecuencias de los acontecimientos protagonizados por la humanidad. Y así explica la formación del imperio acadio a partir de una causa: «la acción del rey Sargón, rey acadio que logró reducir la resistencia de las ciudades sumerias, estableciendo así la supremacía en la región*.

Además de responder a una determinada manera de concebir la historia, los textos no contienen bibliografia actualizada. 11

Un intento de cambiar la metodología de trabajo para los alumnos es la sección dedicada al análisis e interpretación de mapas, de ilustraciones y de fuentes. 12

En un manual de Historia Antigua y Medieval de la editorial Plus Ultra editado en 1981 encontramos indicaciones para los alumnos de cómo deben estudiar y de los errores que deben evitar, con expresiones como no estudies de memoria. Son muy pocas las cosas que requieren memorización. Si estudias con atención y entusiasmo, fijarás rápidamente nombres, fechas y datos. $\$ 13$

Afirmación harto contradictoria donde finalmente se pone el acento en el ejercicio memorístico de acumulación de datos; y se observa a lo largo de todo el libro, en la narración y en las actividades de repaso y fijación, esta ejercitación. Así en el capítulo III que trata sobre la civilización egipcia, una de las preguntas es $i$ Quien fue Nefertiti? 14

A partir de una nueva concepción de la historia con la Escuela de los Annales, los aportes de otras ciencias en este siglo y el estudio de la dinámica social de Fernand Braudel, los historiadores cambiaron sus enfoques, sus categorías de análisis y sus temas. ${ }^{15}$

Cómo esta nueva forma de entender la historia se intenta reflejar en los libros de texto, se visualiza ya en el manual de $\mathrm{AZ}^{16}$ y en mayor medida en el de Santillana (ed. $1988)^{17}$, donde se plantea como objetivo la reflexión de los problemas de los hombres en sociedades del pasado. Objetivo ambicioso que no se cumple en la medida que los temas se tratan a partir de contenidos sólo informativos ${ }^{18}$ y que no escapan al relato político institucional. Sin embargo, en el manual de la editorial Santillana, por un lado las actividades permiten comprender, analizar, comparar, sintetizar, realizar ciertas habili- 
dades como elaborar gráficos y cuadros, (no necesariamente procedimientos en la terminología colliana) ${ }^{19} \mathrm{y}$, por otro, el enfoque de ciertos temas como la información que contienen está más actualizada, como por ejemplo la clasificación de los pueblos del mundo antiguo desde el punto de vista lingüístico. 20

La bibliografia citada para consulta en el área de la historia antigua de Cercano Oriente corresponde a obras o trabajos editados entre las décadas de los años 60 y $70^{21}$. Muchos de ellos como el trabajo de Frankfort, H.; Reyes y dioses 22 , o el manual de Paul Garelli23, son actualmente necesarios para la comprensión de la historia del Cercano Oriente Antiguo, pero sin una adecuada incorporación de las investigaciones y obras generales más recientes ${ }^{24}$, las nuevas interpretaciones quedan restringidas sólo al ámbito de investigación y universitario.

Creo que el problema fundamental reside en que los manuales resumen interpretaciones históricas para hacerlas accesibles a los estudiantes secundarios ${ }^{25} \mathrm{o}$ dan, a menudo, explicaciones simples sobre los cambios sociales y describen las sociedades sin plantear entonces los problemas en su complejidad.

Tomando el origen de los primeros estados, se observa una tendencia general a explicar este tema a partir de la teoría de la conquista o el nacimiento de las ciudades. No se tienen en cuenta otros factores que intervinieron en este proceso, simplificando así los cambios y dificultando a los alumnos su comprensión ya que los conceptos aparecen como entidades abstractas. Así «el estado egipcio se originó cuando el Alto Egipto conquistó al Bajo Egipto en el 3000 a. C., cuando aparecieron las ciudades» o en los últimos manuales aparece la idea del control del agua. Quedaría por integrar entonces los mecanismos que dieron origen a los primeros estados, es decir a la forma más temprana de sociedad de clases; esto no significa de ningún modo la descripción detallada de cada una de las teorías conocidas a través del tiempo ${ }^{26}$. Un primer paso será comprender la evolución de las primeras sociedades a las sociedades estatales, entonces aparecerá claramente cómo llegan estas últimas a constituirse: cómo el poder estaba basado en la creación y control de excedentes agrícolas ${ }^{27}$, entre otros mecanismos que desencadenaron el proceso y que no me detendré a desarrollar aquí.

Queda claro del precedente análisis que, para la enseñanza y aprendizaje de procesos históricos, los antiguos manuales no brindan textos que permitan su interpretación y comprensión.

A partir de los nuevos enfoques de la disciplina y las distintas posturas historiográficas, ¿es posible trasladar esta discusión al ámbito de la escuela secundaria? ${ }^{28} i^{0}$ es posible hacer una síntesis de contenidos que incluya conceptos, procedimientos explicativos e investigativos que sean significativos ${ }^{29}$, (búsqueda inacabada de nosotros los docentes), sin caer en una mera explicación de conceptos y de grandes procesos vacíos de contenido y, por lo tanto, de sentido?

¿Qué dificultades se les presentan a los alumnos para aprender el vocabulario propio de la disciplina y comprender un tiempo y espacio tan remotos? 
Antes de contestar estas preguntas debemos pensar qué queremos que los alumnos aprendan y para qué. Aclarar los objetivos de la enseñanza de la historia nos permitirá definir contenidos y métodos de aprendizaje. El objetivo de la enseñanza de la historia antigua en la enseñanza media es muy distinto al que perseguimos los historiadores interesados en ella, ya que la búsqueda de los docentes será que los alumnos adquieran ciertos conceptos y contenidos problemáticos que le permitan «pensar históricamente 30 . Debemos lograr que desarrollen el pensamiento crítico planteando cuestiones, problemas que les permitan reflexionar sobre sus propias ideas ${ }^{31}$.

En los últimos años se ha sostenido que el alumno no va aprendiendo cosas nuevas mediante una simple acumulación de lo que se le presenta mediante los libros de texto, sino que aplica sus esquemas previos para construir su conocimiento 32 .

M. J. Rodrigo ${ }^{33}$ señala que las diferencias en las epistemologías de partida producen discontinuidades (tipo de fenómenos que cada uno explica, metas que pretende alcanzar, procedimientos de construcción y contexto de construcción) entre el conocimiento del hombre de la calle, el científico y el alumno. Y que las explicaciones cotidianas de los alumnos son difíciles de reemplazar por las explicaciones científicas, ya que el científico intenta descubrir un conocimiento nuevo, cada vez más cercano a la verdad, mientras el alumno intenta reconstruir un conocimiento ya construido por otros.

Según S. Finocchio ${ }^{34}$ «La historia que se enseña no es la de los historiadores (...) Los contenidos de la enseñanza resultan de la "transposición didáctica» es decir del proceso que transforma al conocimiento científico en otro tipo de conocimiento con el objetivo de adecuarlo a las particularidades de la vida escolar» ¿Cuáles serán entonces los puntos de partida para la reconstrucción de un conocimiento nuevo: el conocimiento escolar 35 , que ayude a comprender la sociedad apropiándose de conceptos de la disciplina? ${ }^{36}$

Esto no implicaría trasladar los enfoques históricos actuales al ámbito secundario ${ }^{37}$ sino acercarse a los conceptos de la disciplina. ¿Qué significa acercarse? «No es posible enseñar conceptos sin trabajar la información que da cuenta de los mismos. Comprender determinados conceptos significa comprender el papel de éstos como organizadores de la información. Se trata entonces de que manejen datos y de que, al mismo tiempo, adquieran una lógica que les permita pensarlos (...)».38

La relación estrecha con el tema que nos ocupa es cómo llega esta información a los alumnos, de tal manera que esta *ansiada lógica» sea posible.

Está visto que para el enfoque tradicional, los textos respondieron a la necesidad, en una versión de Escuela Media, de transmitir un conocimiento disciplinar erudito. Necesidad de las editoriales que se han ocupado de que los cambios en los contenidos curriculares no afecten la venta de sus libros. ${ }^{39}$

Por otra parte la creencia tan difundida de que la historia escolar «debería llegar a ser» como la misma disciplina, con sus categorías de análisis, su método, sus interpretaciones, crea una brecha entre la lectura escolar y la bibliografía propia de la disciplina. 
Las nuevas propuestas superan, desde el punto de vista didáctico y metodológico, a las anteriores, pero los textos informativos recurren a explicaciones simples para analizar procesos de la historia antigua. Tal es el caso del manual de la editorial Aique (1993) 40 en el cual se evidencia una clara renovación de las actividades de aprendizaje, coherencia en la selección y organización de contenidos y por primera vez una inclusión de los conceptos dentro del proceso de enseñanza. Pero cuando tomamos por ejemplo las condiciones de la propiedad de la tierra en Mesopotamia observamos que se sintetizan de tal manera las ideas que se cae en una falsa interpretación del tema:

(...) *Tanto el templo como individuos particulares poseían también tierras, pero el estado podía quitárselas si no las trabajaban correctamente. Había severas penas y castigos para quienes cometían ese delito.» (...) Existe una discusión acerca de la tierra del templo en Mesopotamia (Diakonoff, op. cit.; Falkenstein, A., La ciudad templo súmera en Cahiers d' historie mondiale I No 4 (1954), pp. 784-802. Además se reduce el problema a «el trababajo correcto" de la tierra ${ }^{41}$.

Entender que el alumno, a diferencia del científico, no mantiene la ilusión de descubrir cosas nuevas cuando las ve escritas en el libro, perdiendo protagonismo en el proceso constructivo ${ }^{42}$; y que estudiar historia en el colegio secundario supone en primer lugar adquirir los elementos necesarios para comprender la realidad socioeconómica y política presente y operar sobre ella 43 ; nos llevará a encontrar a partir de situaciones concretas, problemas y lapsos temporales más amplios y complejos ${ }^{44}$, un camino para construir la enseñanza de una historia *que promueva la reflexión crítica sobre la realidad social $(\ldots) * 45$

Esta afirmación general nos enfrenta con distintos problemas puntuales:

a) La idea de plantear «situaciones problemáticas para que el alumno reconozca la nececesidad de relacionar el problema en cuestión con aquello que ya conoce» y así se coloca en el lugar de protagonista. Esta es la propuesta del libro «Pensar, descubrir y aprender» de Alicia Camilloni y Marcelo Levinas 46 quienes proponen una metodología que se centra en el abordaje de situaciones que introducen conceptos de las ciencias sociales. Esta manera de empezar a pensar el estudio de las sociedades es una tarea creativa de construcción de conceptos y organización de los mismos: en palabras de un alumno «para que nosotros pusiéramos las soluciones como si estuviéramos ahí».

Pero, cuando el alumno se inicia en algún estudio sistemático de la vida de las sociedades debe dar a los conceptos un significado más riguroso y preciso ${ }^{47} \mathrm{y}$, además, como los mismos autores expresan la selección de los contenidos resulta arbitraria en las diferentes actividades 48 .

Los docentes debemos aceptar el desafío de contribuir a la reconstrucción por parte de los alumnos de un conocimiento de la historia donde pueda visualizar sistemas de producción, relaciones económico-sociales entre grupos humanos, y comprender la dinámica social. 
b) No es el objetivo de este trabajo el análisis de la noción de tiempo histórico. Pero si hablábamos de una historia de los manuales descriptiva, que se preocupaba solamente por el tiempo de las fechas y datos, de los acontecimientos; debemos señalar que la importancia del concepto de tiempo a la «hora de construir un currículo" 49 y por lo tanto de la organización de los temas y selección de textos de un manual es imprescindible 50 .

El diseño curricular más generalizado ha sido el diseño cronológico ${ }^{51}$. Se ha planteado entonces la necesidad de una base cronológica secuencial o la historia perdería su especificidad como disciplina ${ }^{52}$.

A partir de sus investigaciones, Asensio, Carretero y Pozo ${ }^{53}$ consideran que con el aprendizaje memorístico de fechas se le va proporcionando al alumno un marco temporal, aunque sea fragmentario y escasamente comprensivo. Y consideran que «con estrategias educativas donde se prime la comprensión, si no la conseguimos, corremos el peligro de no proporcionarle al alumno ningún marco». La idea que presentan es la comprensión de nociones temporales y no la comprensión de procesos de tiempo donde la información, (dícese datos, fechas) adquiere un significado a partir de los conceptos envueltos en la lógica que explica las relaciones entre los grupos sociales a través del tiempo.

Tal vez los alumnos de primer año presentan dificultades para la comprensión de la continuidad y el cambio social ${ }^{54}$, pero no existen hasta el momento estudios sobre el tema ${ }^{55}$, y sí podemos afirmar que pueden recrear imágenes sobre los procesos históricos no estáticos y con rupturas, y pueden integrar, además, a la historia política.

A partir de las investigaciones realizadas en diversos ámbitos de la psicología sobre los procesos de aprendizaje de las nociones sociales y los aportes de la didáctica estamos en un camino de reflexión constante sobre nuestra práctica docente desde la perspectiva didáctico metodológica.

¿Cómo acercarnos al campo disciplinar en esta reflexión que nos atañe a todos?

Es nuestra tarea como docentes organizar los contenidos en un cuerpo de conceptos para poder explicarlos en una lógica. Sabemos que los manuales por sí solos no van a colaborar en esta difícil tarea, sobre todo en lo que se refiere a los textos. Debemos entonces plantear estrategias para que los alumnos puedan reflexionar a partir de la información que nos brindan los manuales y además seleccionar textos alternativos para que los alumnos puedan comprender los ejes fundamentales de los procesos históricos que tuvieron lugar en la antigüedad.

Dentro de una secuencia cronológica que nos permita dar un marco referencial y particular en cada caso, es fundamental tomar como ejes cuestiones tales como la formación de los primeros estados (primarios y secundarios), la fundamentación ideológica de los mismos, las formas de propiedad y posesión de la tierra, la circulación económica y los intercambios, las relaciones y conflictos sociales, la relación entre nómades y sedentarios, y las diferencias entre las ciudades estado y estados territoriales. La reconstrucción de la historia de las sociedades y estados necesita del lineamiento histórico general y esto implica a otras cuestiones como la identidad lingüística de los 
grupos que protagonizaron la historia antigua, las relaciones entre los pueblos y los movimientos migratorıos, las características del medio ambiente físico y los asentamientos y las relaciones entre los grandes estados.

\section{Notas}

(1) ¿Cujal es entonces el beneficio para los historiadores de estudiar la invención de la tradición? En primer lugar, porque son indicadores de problemas que quizás de otro modo no hubieran resultado visibles. $y$ de desarrollos que de otra manera serian dificiles de identificar y de asignarles una fecha (...) En segundo lugar. arroja luz sobre ia relación de los seres humanos con su pasado, y por io tanto sobre la tarea misma del hustoriador (...) Traducción de Hobsbawn, Eric. *Introduction: Inventing Traditions* en Hobsbawn, E. and Ranger. T. (ed.) The invention of Traditions. Cambridge University Press, 1983. Discurso histórico-1-Facultad de Filosofia y Letras. UBA. De por quė el tratamiento del tema comienza por la tradición.

(2) Ibañez. C.. Historia Antigua y Medieval. Ed. Troquel S.A.. Buenos Aires. decimosexta edición. 1965.

(3) Drago. A. L.. Historia Antigua y Medieval, Ed. Stella. Buenos Aires. primera edición. 1966.

(4) Tuñon de Lara, M. ¿A qué llamamos historia?, en ¿Por qué la historia?. Salvar Editores. Madrid, 1985.

(5) Tunon de Lara. M., op. cit., 1985.

(6) Drago. A. L., op. cit., p. 55.

(7) Ibañez. C. op. cit., p. 50.

(8) En el caso de la civilización mesopotámica se describen las caracteristicas dei palacio y el templo desde la época de las ciudades estado súmeras a la época de domınación asiria. pero no su transformación o sus conflictos a través del tiempo. Romero. J. L.. Historia de la Antiguedad y de la Edad Media. Editorial Estrada y Cía S. A..

(9) Rozada Martinez sen̂ala que esta "Historia más explicativa no ha dejado de plantear problemas de aprendizaje. . Rozada Martinez. J. M., La enseñanza de las ciencias sociales: Una cuestión «indisciplinada», necesariamente, Aula de Innovación Educativa $N^{\circ} 8.1992$, p. 7.

(10) A. Zeta ed.. Historia. Primer Curso. Contenidos Mínimos. $6^{\circ}$ edición. 1988.

(11) La bibliografia citada para los temas del Cercano Oriente Antiguo por el manual de A. Zeta corresponde en su mayoria a obras generaies y no han utilizado material actualizado. ejemplo de ello son: -André Aymard. Las civilizaciones imperiales del Próximo Oriente. en Crouzet. M.. Historia General de las Civilizaciones; -Kitte!, Rodolfo, Los pueblos del Oriente Anterior; -Contenau. G. Antiguas civilizaciones del Asia Anterior.

(12) Ejemplos de mapas son el que señala las migraciones indoeuropeas y semitas y sobre el Nilo egıpcio en lo que respecta a ilustraciones y fuentes han seleccıonado varias interesantes como las pinturas y esculturas del antiguo Egipto que nos informan sobre la vida cotidiana. A. Zeta ed., op. cit., pp. $37 ; 19$ y 25 respectivamente.

(13) Suárez. M. E., Ambrós, A. T., Historia Antigua y Medieval. Ed. Plus Ultra, Buenos Aires, 1981, p. 18.

(14) Suárez. M. E.. Ambrós. A. T., op. cit.. p. 123.

(15) Barietta. A. M. de Amezola. G.. Esquizohistoria e histortofrenia. Del secundario a la carrera de Historia Y vuelta al secundario. Entrepasados. Revista de Historia. Año II No 2, 1992. p. 92.

(16) A. Zeta ed.. op. cit.

(17) González. A. S.. Jáuregui, A. P. Rodriguez, I. M. . Historia 1, Ediciones Santillana, S. A., Buenos Aires. 1988.

(18) - Para que los datos y los hechos cobren significado. los alumnos deben disponer de conceptos que les permitan interpretarios.- Los datos sólo tendrän stgnificado si se siruan en el marco de un sistema de conceptos.(...) -Para aprender un cuncepto es necesario establecer relaciones significativas con otros conceptos. Cuanto más entretejida esté la red de conceptos que posec una persona en un área determinada, mayor será su capacidad para establecer relaciones significativas y por tanto para comprender los hechos propios de esá árean. Pozo, J. I., El Aprendizaje y la Enseñanza de Hechos y Conceptos, en: Coll, C. y otros. Los Contenidos de la Reforma. Enseñanza y Aprendizaje de Conceptos, Procedimientos y Actitudes, Aula XXI. Santillana, 1992. pp. 22-23. 
(19) En la práctica. como lo senalan Coll y Valls. se trabajan en forma conjunta procedimientos y conceptos. El procedimuento puede lleģar a ser un conjunto de actuvidades para adquirir los contenidos pero sólo cuando el profesor to convirtese en ohjeti directo de aprendizaje. Coll. C. y Valis, E.. El Aprendizaje y la Enseñanza de tos procedimuentos, en: Coil. C. y otros. op. chl. pp. 98-99.

(20) González. A. S., Jâuregut. A. P.. Rodriguez. I. M1.. op. cit.. pp. 36-37.

(21) Algunos de los tímlos de la hibliogratia citada pars Antiguo Onente son: -Drioton. E.: Vandier. J.. Historia de Egipto. Ed. Eudeba. Buenos Aires. 1973: Bottero. J.: Cassın. E.. Vercouter. J.. Los imperıns del Antuguo Onente. Madrid. Ed. Siglo XXI. 1975. Schmokel. H.. El pais de los súmeros. Ed. Eudeba. Buenos Aires. 1965. Wilson. J. A.. La cultura egipcia. F. C. E.. México, 1972. Gonzảlez, A. S.. Jáuregui. A. P.. Rostriguez. 1. M. op. cit

(22) Frankfort. H., Reyes y dioses. Ed. Biblioteca de la Revista de Occidente. Madrid. 1976.

(23) Garelli. P.. El proximo Oriente asiatico. Ed. Labor. Barcelona. 1974.

(24) -Butzer. K.. Early hydraulic civilization in Egypt. 1976.

-Carneiro, R. A theory of the Origin of the State. en Science 169 (1970), pp. 733-738.

-Claessen. H.. The internal dinamics of the early state. en CA (1984). pp. 365-379.

-Diakonoff.I. Estructura de la sociedad y el estado en el Dinástico Temprano en Sumer, vol. 1 (1974). pp. 3-16.

-Godelier. M. Las sociedades precapitalistas. Laia. Barcelona. 1977.

-Hobsbawn. E.. Formaciones ezonómicas precapttalıstas. Ed. Crítica. Barcelona, 1979.

-Janssen. J. J.. El rol del templo en la economia egipcia durante el imperio nuevo. en Lipinski ed. State and Temple in the Ancient Near East. 1979. vol. 2. pp. 505-515.

-Kemp. B.. El Antiguo Egipto. Anatomía de una civilización. Ed. Crítica. Barcelona. 1989.

-Kupper. J. R.. El papel de los nomades en la historia de la Mesopotamia Antigua. en JESHO II, parte II (1959). pp. 113-127.

-Liverani, M. . Contrastes y confluencias de coneepciones politicas en la época del Amarna, en RA 61 (1967), pp. 1-18.

-Polanyi, K., Comercio y mercado en los imperios antiguos. Ed. Labor, 1976.

-Redman. Ch., Los origenes de la civilización. 1990.

-Trigger. Historia del Egipto Antiguo. Ed. Critica. Barcelona. 1985.

Estos son sólo algunas de las recientes obras generales y trabajos de investigación sobre temas fundamentales en la historia de las prımeras civilizaciones. Muchos de éstos fueron publicados con anterioridad a la edición de los manuales y no han sido consultados, como por ejemplo el libro de Polanyi sobre el comercio en la antigüedad o el artículo de Mario Liverani sobre las relaciones entre los imperios en el segundo milenio. Para los docentes dedicados a la enseñanza media resultaría imposible estar al tanto de estas cuestiones. dificultad que se salvaría si los manuales proveveran un enfoque de los temas con bibliografía más completa $y$ actualizada.

(25) Barletta. A. M., de Amezola. G.. op. cit., p. 93.

(26) La preocupación por explicar los origenes del estado y la civilización la encontramos desde los filósofos clásicos griegos y romanos. los teóricos de la ilustración europea. hasta la actualidad. Existen tantas teorias que sería imposible desarrollarias en este espacıo sólo me limitaré a mencionar algunas: Teoría del conflicto (Spencer). Teoria de la conquista (Ibn Jaidún). Teoria Hidraúlıca (Wittogel), Teoria de la circunscripción geográfica (Carneiro). Teoria del control de la producción y de la redistribución o de la urbanización (Gordon-Childe), Teoría demográfica. Teoría del crecimiento y desarrollo, Teoría del Intercambio y la Teoría del materialismo histórico.

(27) Estos excedentes mantenian un estilo de vida de elite que se distinguia por su lujo. la creación de arte monumental. y otros simbolos de status. Trigger. B.. Early Civilizations. Ancient Egypt in Context. The American University in Cairo Press. 1993. Parte uno. Cabria preguntarse entonces: ¿Cómo llega esta sociedad a esta estratificación? ¿Cuáles son las relaciones entre los grupos antes de originarse el estado?

(28) Barletta. A. M., de Amezola. G.. op. cit., p. 91

(29) Coll. C. y Valls, E., El Aprendizaje y la Enseñanza de los procedimientos, en: Coll, C. y otros, op. cit., pp. 106-117. 
(30) Como dice J. L. Romero -pensar históricamente* *consiste principalmente en acostumbrar a examinar el revés de la trama-(...), es importante que se enuncien los hechos políticos. pero (...) lo importante es que se le dé al adolescente algo más: algo que lo incite a buscar qué hay detrás del puro episodio. Esto supone que ios profesores y los autores de texto partan del principio de que el análists histórico debe referirse a procesos y no a hechos.. Romero. J. L.. Enseñar historia es posible, en: ¿Se enseña en la Argentina la historia real del pais?. Revista Crisis $N^{\circ} 8,1973$. La cursiva es de la autora.

(31) Pagés, J., Una unidad didáctica: "Trabajo, paro y ocio ". De la teoría a la práctica. Cuadernos de Pedagogía $\mathrm{N}^{\circ} 178$, Barcelona. 1990, p. 22 ..

(32) Carretero, M.: Asensio. M., La enseñanza de la Ciencias Sociales: aspectos cognitivos y psicopedagógicos. Temas acruales sobre psicopedagogía y didáctica. F. Huarte. Ed. Marcea, pp. 205-221.

(33) Rodrigo. M. J.. El hombre de la calle, el cientifico y el alumno: ¿un sólo constructivismo o tres?. Investigación en la Escuela No 23. (1994), pp. 7-15.

(34) Finocchio. S. Enseñar Ciencias Sociales, II. Criterios para revisar la enseñanza de las Ciencias Sociales. Troquel Educación. Buenos Aires, 1993. pp. 15-31.

(35) Rodrigo. M. J.. op. cit. . (1994). pp. 10-13. “Consideramos el conocimiento escolar como el conocimiento que se elabora en la escuela que, (...) aunque tiene como marco de referencia el conocimiento cientifico. no es un conocimiento científico en si, sino una elaboracion de este conocimiento que se ajusta a las características propias del contexto escolar. (Cubero y García, 1994) en: Gil Pérez. Daniel, Relaciones entre el conocimiento escolar y conocimiento científico. Investigación en la Escuela N०23 (1994), pp. 17-32.

(36) Como lo explica Silvia Finocchio -podrán acercarse a los conceptos de la disciplina en tanto puedan poner en duda enriquecer $y$ hasta deshacerse de las ideas anteriores-, op. cit., p. 26.

(37) Barletta, A. M., de Amezola, G., op. cit., p. 91.

(38) Finocchio, S., op. cit., p. 22.

(39) Barletta, A. M.. de Amezola. G.. op. cit.. p. 93.

(40) Alonso. M.: Elisalde. R.: Vázquez, E.. Historia. La Antigüedad y la Sociedad Feudal, Aique Grupo Editor S. A., $1^{\circ}$ Edición, 1993.

(4i) Alonso, M.: Elisaide. R.: Vázquez. E., Historia, op. cit., p. 88.

(42) Rodrigo, M. J., op. cit., (1994).

(43) Informe de avance sobre el desarrollo del Proyecto: ¿Diagnóstico sobre los programas vigentes de la asıgnatura Historia. Bases y propuestas para su posible reformuiación.. Departamento de Ciencias del Hombre. Colegio Nacional Rafael Hernandez. Universidad Nacional de La Plata. Año 1992, p. 1.

(44) Informe «Diagnóstico sobre los programas vigentes de la asignatura Historia (...)”, op. cit., p. 15.

(45) De Blas. Patricio. Reforma de las ciencias sociales en Bachillerato. En Apuntes de Educación. Madrid $N^{\circ}$ 19. 1985 .

(46) Camilloni, A.; Levinas. L.. Pensar, descubrir y aprender, Ed. Aique, Buenos Aires, 1989.

(47) Boulding, K. The Task of the Teacher in the Social Science, en Effective College-Teaching, William H. Morris Ed., American Council of Education, 1970, citado por Camilloni, A.: Levinas, L., op. cit., p. 9.

(48) Camillon, A.; Levinas, L., op. clt., p. 21.

(49) Carretero, M.; Pozo, J. 1.; Asensio, M. (Compiladores). La enseñanza de las Ciencias Sociales. capitulo V: La comprensión del riempo histórico. 1989, pp. 103-137.

(50) Braudel considera que - El historiador no se evade nunca del tiempo de la historia: el tiempo se adhiere a su pensamiento cono la tierra a la pala del jardinero- (...) Braudel. F., La historia y las ciencias sociales, Alianza Editorial. Madrid, 1974.

(51) Carretero. M.; Pozo, J. 1.: Asensio. M., La comprensión f...). p. 107.

(52) Informe "Diagnóstico sobre los programas vigentes de la asignatura Historia (...)», op. cit., p. 13.

(53) Carretero. M.; Pozo. J. I.; Asensio. M.. La comprensión (...). p. 132.

(54) J. Le Goff en la Entrevista acordada a Silvia Perez Ringuelet en diciembre de 1988, en la Ecole des Hautes Eudes en Sciences Sociales afirma que «la historia braudeliana y en consecuencia en la segunda fase de los Annales se olvidaron que la historia es cambio y movinientom.

(55) Carretero. M.: Pozo. J. I.: Asensio. M.. La comprensión (...). p. 127. 